Lehtovaara, J., Seppänen, O., and Peltokorpi, A. (2019). "Improving the Learning of Design Management

Operations by Exploiting Production's Feedback: Design Science Approach" In: Proc. 27th Annual Conference of the International. Group for Lean Construction (IGLC), Pasquire C. and Hamzeh F.R. (ed.), Dublin, Ireland, pp. 25-36. DOI: https://doi.org/10.24928/2019/0143. Available at: 〈www.iglc.net>.

\title{
IMPROVING THE LEARNING OF DESIGN MANAGEMENT OPERATIONS BY EXPLOITING PRODUCTION'S FEEDBACK: DESIGN SCIENCE APPROACH
}

\author{
Joonas Lehtovaara $^{1}$, Olli Seppänen ${ }^{2}$, and Antti Peltokorpi ${ }^{3}$
}

\begin{abstract}
This study examines the development and implementation of a learning process in a contractor's design management unit. The purpose is to gain knowledge on how learning can be turned into a standardized process and of methods of accelerating the learning in a design management unit by exploiting the feedback received from the production.

The research took a design science approach, which consisted of a diagnosis of the present situation, testing \& development of the formulated process, and analysis \& generalization of the results. The diagnosis comprised a literature review, interviews, and active observation, while the testing phase included an intervention where the process was tested and further developed.

The results indicate that while the relevancy of continuous learning is well recognized, construction organizations are incapable of effectively exploiting the best practices of knowledge management. To overcome weaknesses related to the inefficient learning practices, organizations should focus on balancing the operational and strategical viewpoints of learning, emphasize learning from failures and implement project-based communities of practice into an organization's operations.

The study has implications for more standardized and balanced learning processes in contactor operations. It also provides knowledge of ways of taking a design science approach effectively in construction management research.
\end{abstract}

\section{KEYWORDS}

Design management, organizational learning, knowledge management, design science

1 Doctoral Candidate, Department of Civil Engineering, Aalto University, Finland, joonas.lehtovaara@aalto.fi

2 Professor of Practice, Department of Civil Engineering, Aalto University, Finland, Olli.seppanen@aalto.fi

5 Assistant Professor, Department of Civil Engineering, Aalto University, Finland, antti.peltokorpi@aalto.fi 


\section{INTRODUCTION}

To increase productivity in construction, organizations and especially design management operations should exploit the knowledge created in previous projects, and particularly the knowledge created in the production phase, more effectively. Carrillo \& Chinowsky (2006) reason that the most recent knowledge of the design solution's constructability and correctness - in other words, the most recent knowledge regarding the requirements for flow-efficient and value-adding designs - is held by the individuals working in production. They argue that for construction companies to increase their productivity, more efficient learning processes through the coupling of production and design operations are essential.

The importance of effective learning through coupling has been reported in construction management research widely. Several studies consider knowledge management and effective learning among the most important core competencies of construction companies (e.g., Dave \& Koskela, 2009, Henderson et al. 2013, Carrillo et al. 2013). In addition, Giridhar et al. (2018) suggest that knowledge management plays a vital role in implementing lean culture effectively into organizations. Even though the identification, collection, analysis, storage and reuse of tacit and explicit knowledge from projects has been recognized as a pertinent part of the learning of design operations, the nature of the project-based industry sets barriers for development. Dave \& Koskela (2009) mention that, for example, the fragmentation of the project organizations, constant rush, and reluctance towards radical development hamper effective learning in and between projects. These barriers have led to reinventing the wheel all over again, solving problems reactively and ignoring the deeper root causes, while the lessons from other projects are not transferred into following projects, or to design solutions in the following projects. Henderson et al. (2013, Figure 1) argue that the lack of proactive, double-loop learning from an actor's own mistakes is one of the fundamental reasons for poor productivity development.

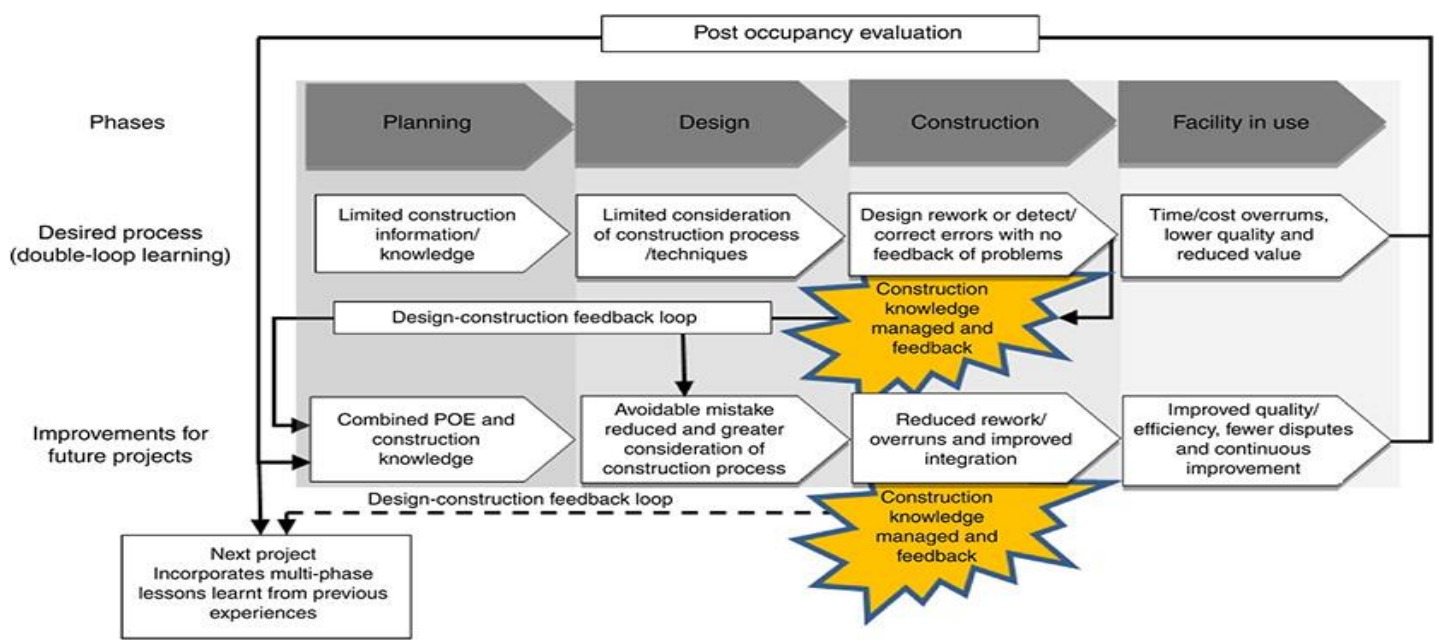

Figure 1: Desired double-loop learning through a design-construction feedback loop (Henderson et al. 2013) 
The reactive touch to learning reflects the inefficient implementation of new learning processes, which have been criticized for their inefficiency to drive change. Eriksson (2013) argues that research on project-based organizations has centred only on visible problems at hand and asking what should be done, instead of focusing on how to concretely solve the problems. Holmström et al. (2009) suggest that to overcome this inefficiency in management research, researchers should take a more active role in designing the solutions instead of only observing from a distance. In a similar vein, some researchers (e.g. AlSehaimi et al. 2012 and Azhar 2009) have suggested implementing experimental and active research methods, such as design science research, into construction.

Therefore, there is a certain need for designing but also for concretely implementing more effective learning processes into the construction design operations. The goal of this study is to develop a concrete construction design learning process by an experimentative design research approach while answering the following research question: How can the learning of design management operations in a construction company be accelerated through feedback acquired from production?

\section{RESEARCH DESIGN}

Design science research is an iterative and experimental approach in which the development is conducted in tight, reciprocal collaboration between theory and practice (Wang \& Hannafin 2005). This study follows the approach designed by Holmström et al. (2009) and comprises three steps: 1) the diagnosis of the current situation and development of the preliminary learning process from the basis of the diagnosis; 2) testing and development of the process and 3) generalization of the findings and demonstration of a theoretical contribution. The research process is presented in Figure 2.

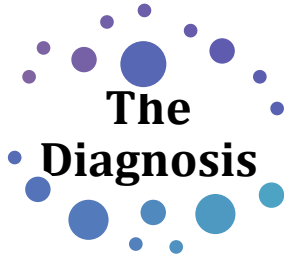

- The diagnosis of the current state \& development of the preliminary learning process - Literature review, interviews, observation

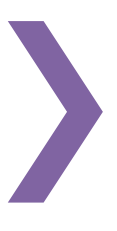

Testing and development

- Testing and development of the process in collaboration with the target company

-Collaborative development, workshop, validation

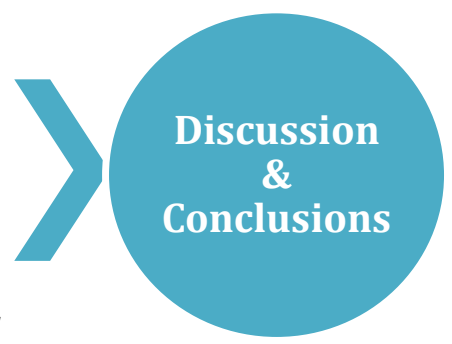

Figure 2: The structure of the design science research

First, in the diagnosis phase, the authors attempt to find and describe the current state of the observed case. The observations are combined with the literature review, to gain holistic but also specific understanding of the situation. The diagnosis follows an abductive process (Kovacs \& Spens 2005), meaning that while the literature review shapes the 
interviews and observation, the empirical results also direct the literature review reciprocally. Thus, the steps in diagnosis phase are highly intertwined, and presented in symbiosis in the paper. The adequate learning process is developed through the diagnosis. Second, in the testing and development phase, the process is tested and further developed through collaboration with the target company's personnel, including the preparation, testing in workshop, and analysis of the tested process. Third, in the discussion and conclusion phase, authors address whenever the research question was answered properly, and attempt to provide avenues for future research.

The development of the learning process is inspected from the viewpoint of a Finnish general contractor's residential unit. The selection of the case was impacted by theoretical and practical interests. As a relatively typical residential construction unit, the results are generalized in a more straightforward manner, whereas the target company's interest in development offers a concrete premise for the active research design approach.

\section{THE DIAGNOSIS}

The diagnosis of the current state consists of a review of current knowledge management and construction operations management literature, nine semi-structured theme interviews of personnel working in company's residential construction unit, and active observation of the company's practices for four months. The adequate learning process for the company's design management operations is developed through the diagnosis, combining the existing literature as well as analysis of the target company's current practices.

\section{KNOWLEDGE MANAGEMENT IN CONSTRUCTION OPERATIONS}

Construction can be considered a knowledge-intensive industry, where projects and organizations constantly create a vast amount of information and knowledge. However, the complexity of projects and the previously mentioned barriers to industrial development have created a situation where a vast amount of knowledge is continually lost and an opportunity for learning is missed (Dave \& Koskela 2009). Almeida \& Soares (2014) argue that this opportunity is lost because a large amount of created knowledge is typically structured to directly benefit only the project at hand. While the approach creates more tangible gains for the current project, information is usually dispersed into different locations and loses its potential for efficient learning and exploitation after the project ends. To effectively prevent knowledge dispersion, Dave \& Koskela (2009) note that organizations should focus more on long-term development and process-based knowledge management, instead of investing resources only in projects. To enable effective knowledge sharing and learning through the organization, an organization needs a tangible knowledge management strategy.

An organizational knowledge management strategy can be based on the balance between two different approaches. Personalization, a human-based view, emphasizes the meaning of tacit knowledge and is usually present in small and agile organizations. In contrast, codification, a technology-based view, describes an approach where knowledge is managed through systems and documents and the knowledge is mainly explicit (Hansen et al. 1999). Even though organizations should determine which approach is dominant in 
which processes, it does not mean that organizations should focus solely on one approach. Lee \& Choi (2003) with Mäki (2008) argue that the knowledge management strategy is implemented most effectively when the instruments are determined on the sub-process level, which enables the link between an organization's strategy and tangible operational actions.

Expert interviews yielded observations similar to those in literature. If the learning processes are invisible in the organization, the knowledge management strategy is hard or even impossible to implement in operations. Mäki (2008) states that this is common for organizations, and an unclear link between the strategy and the operational learning processes constitutes one of the major barriers for learning. Also, communication between personnel in production and in design operations should be more efficient and more structured to enable continuous learning. The experts reported that communication is often minimized in the hectic project environment if the communication is not absolutely necessary.

\section{LEARNING PROCESSES IN CONSTRUCTION}

In the context of project-based organizations, learning from projects is realized through three concrete steps: 1) collection of information and knowledge created in projects, 2) filtering and analysis of collected information and knowledge, followed by 3) storage of analyzed knowledge (e.g. Carrillo et al. 2013, Dave \& Koskela 2009). The usefulness of the definition is based on its tangibility and the ability to present the stages with three knowledge management frameworks: the SECI model (Nonaka \& Takeuchi 1995), the 4I model (Crossan et al. 1999), and Blackler's (1995) five types of knowledge. Although it is illustrative to inspect these processes in isolation, it should be kept in mind that these processes are always somewhat connected and intertwined.

\section{Creation and collection of information and knowledge}

The creation and collection of knowledge can be examined through the SECI-model (Nonaka \& Takeuchi 1995). The SECI model portrays knowledge creation as a continuous cycle, where the project knowledge emerges through the steps of socialization, externalization, combination, and internalization, as the personnel in production experience, share, combine and reuse the knowledge constantly.

To be useful for the organization, the knowledge that is created in the production phase should also be effectively collected. The process should include the collection of both explicit and tacit knowledge, and the process needs to be connected to the broader knowledge management strategy. Also, the process should be connected to daily activities by implementing concrete tools for knowledge creation as well as collection. Hari et al. (2004) argue that in order for data collection to be meaningful and worthwhile, the implemented tools should promote accessibility and objectivity, and the usefulness of the collected knowledge should be made visible for individuals.

The diagnosis phase revealed that the target organization focused too much on collecting explicit information, and the entire process of learning was too narrowly centred on codification. Ignorance of the accessibility and usefulness of the collected knowledge demonstrated a sub-optimal process, in which the created knowledge did not systematically 
lead to further actions. Several studies (e.g., Hari et al. 2004, Kamara et al. 2002) suggest that this barrier is generally present in construction organizations. To develop the process, Hari et al. (2004) argue that the collection of tacit and explicit knowledge should be more effectively balanced.

\section{The analysis of collected information and knowledge}

Crossan et al. (1999) find that learning in organizations occurs through a knowledge flow between individuals, groups and the entire organization, which can be expressed through the 4I model of learning. They argue that even though every step of the flow is important for learning, the most effective knowledge creation takes place within the group level, where the knowledge from the projects is analyzed and shared with the organization in a reusable way. For project-based organizations, group-level learning can be enhanced by implementing so-called project-based communities of practice (Lave \& Wenger 1998 with Lin \& Lee 2012). In these communities, experts working within the same area of interest (but not necessarily within the same projects), share their knowledge created in the projects, while analyzing the knowledge and simultaneously sharing it with the organization.

In an optimal setting, project-based communities of practice operate freely but at the same time, within a structure that enables continuous filtering and analysis of the created knowledge. When operated effectively, the knowledge flows through the communities and across project boundaries in both codified and personalized form (Lin \& Lee 2012). For effective implementation of the communities of practice, the culture of learning from failure was recognized as one of the most contributing factors, in both literature (e.g., Eriksson 2013, Cannon \& Edmondson 2005) and in the expert interviews.

\section{The storage of the analyzed knowledge}

The knowledge analyzed and shared also needs to be stored. Blackler (1995) states that knowledge can be stored in an organization in five different forms: embrained (conceptual and cognitive abilities, ability to form and visualize knowledge), embodied (know-how, ability to apply knowledge), encultured (collective and shared understanding), embedded (knowledge regarding resources, routines and roles) or in encoded (formal, codified information) form. Embrained, embodied and encultured knowledge can be stored as personalized knowledge, whereas embedded and encoded knowledge are stored in a codified form.

The balance between personalization and codification should be considered also in the storing phase. Digital tools offer a possibility to store codified and distribute personalized knowledge effectively, once the entity of implemented tools is in balance and their usage is easy and meaningful for the employees (Ruikar et al. 2007, also addressed by several interviewees). The interviewees greatly emphasized that the analyzed knowledge should be structured more systematically. For codified knowledge to be reusable, it should be clear where knowledge is stored and whenever it is updated. Also, the personalization of stored knowledge should be emphasized. The interviewees argued that the know-how and rationalization between different design solutions should be presented, so the following projects could apply the knowledge without questioning its validity. Personalized storage could also increase transparency and trust within an organization. 


\section{THE PRELIMINARY LEARNING PROCESS}

On the basis of the conducted diagnosis, a learning process for the design management operations was developed. The main objective for the 3 -step process was to continuously improve the company's design solutions on the basis of feedback acquired from production, while the knowledge flows through an organization in personalized and in codified form. Also, several key requirements for the learning process were identified, taken into account in the testing phase:

- The vocabulary and process components applied should be clearly determined and presented while connecting the strategic and operations-level actions.

- The balance between personalization and codification should be ensured in every process step.

- The process should be accessible, easy-to-use and lightweight. Also, the collection and storage of information and knowledge should be as automated and standardized as possible.

- The project-based communities of practice should enable a space for effective learning from failures and root causes. The community should be supported by an experienced process owner and facilitator.

\section{TESTING AND DEVELOPMENT}

The learning process was iterated and implemented in the unit's operations through an intervention which included preparation, testing, and analysis of the tested process. The preparation of the testing was achieved in collaboration with the unit's key personnel, including the design manager and the unit manager. For testing, one design-specific problem was identified in the feedback acquired from production: the design process of acoustic and fireproofing connection details in drywalls separating apartments (Figure 3). Production personnel raised the issue that because these design solutions are rare (most of the dividing walls between apartments are precast concrete in Finland), they are not automated in the design process, which creates a risk for faulty designs and subsequently also for costly rework. The production engineer suggested that the connection detailing process should be automated by the design management operations.

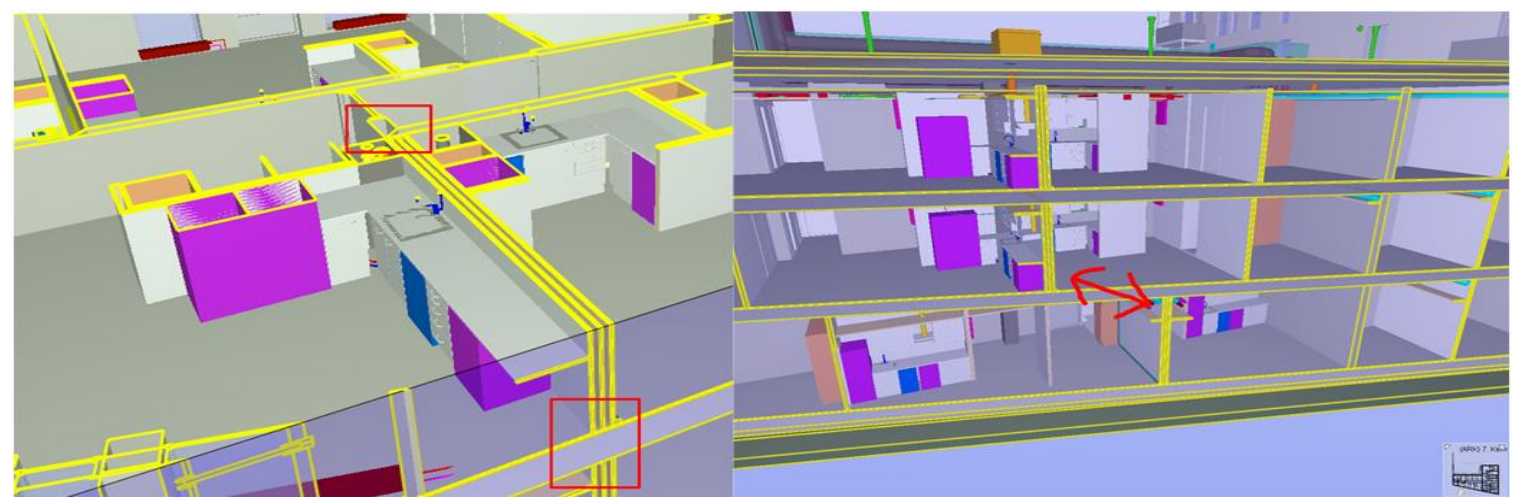

Figure 3: Dividing wall presented in the BIM-model 


\section{1) COLlection OF THE INFORMATION}

The site and project engineers collected more detailed information regarding the received feedback, in both codified and personalized form. They also filtered the information in concise form before assigning it for analysis in the community of practice. The filtered information consisted of basic information regarding the case, feedback from production, as well as preliminary risk and cost analysis. In the collection phase, the goal was to ensure that the community of practice would obtain a tangible problem with sufficient information, but also to ensure that the information was already filtered so that the community could focus on the relevant issues.

\section{2) ANALYSIS: THE COMMUNITY OF PRACTICE}

The meeting of the project-based community of practice was implemented as a workshop which consisted of four stages: 1) site visit, 2) introduction and discussion, 3) creation and root cause analysis, and 4) development of further actions and concrete steps for implementation. Westerlund (2007) argues that these steps are vital for creating a mutual understanding of the problem, for collaborative and creative working, as well as for concretely implementing the results in action. The composition of the community was kept concise (seven key persons of the unit who were responsible for designs), including an experienced facilitator who ensured that the workshop followed the structure, but at the same time, provided a safe space for the community to openly share their ideas.

\section{3) STORAGE OF THE ANALYZED KNOWLEDGE}

After the workshop, the decided actions were stored in the organization's processes in both codified and personalized form. The design manager ensured that the developed solution, including information for implementation, was added to the unit's design library. He also ensured that the additional actions, such as updating internal risk analysis and cost models were adequately completed. In addition to storing the information in documents, the storage also included making the solution visible in the organization, which aimed to reinforce the culture of learning and transparency. The project engineer informed the organization about the new solution through the organization's intranet and provided brief feedback to the project where the feedback was acquired.

\section{ANALYSIS AND FURTHER DEVELOPMENT OF THE LEARNING PROCESS}

After the testing, the process was analyzed with the design and unit managers. The implementation was viewed as a success. The testing also accomplished its initial goal, which was to tackle a specific problem regarding the design by exploiting the feedback from production.

For further development of the process, several actions were proposed. The most important actions concerned process fluency. The process should be as streamlined as possible to minimize the time spent by the participants, and the process steps should be standardized to effectively implement it for another company's internal communities. The constant rush and unclear learning processes were determined as barriers also during the testing phase. The process was further developed to be optimally streamlined and more easily adopted by determining clearer instructions and process steps. Also, a document 
template for easier implementation and education were created. The document followed partially 'A3 problem solving template' (Shook 2008), which is commonly utilized in lean management. Even though the learning process was developed from the basis of knowledge management literature, the problem-solving process shares certain similarities with principles of lean. The core principles of the updated learning process are presented in Figure 4.

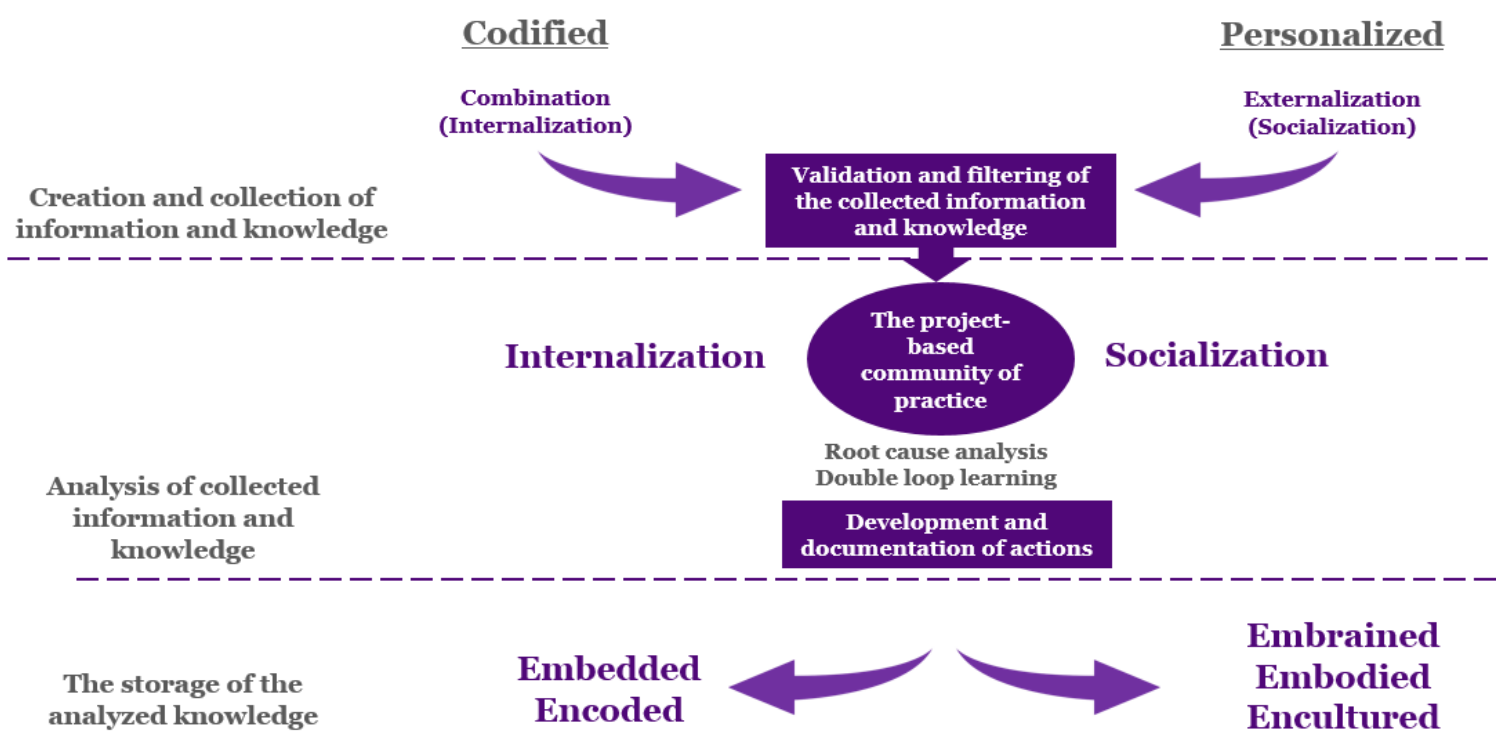

Figure 4: The developed learning process

\section{DISCUSSION}

The research question, "how can the learning of design management operations in a construction company be accelerated through feedback acquired from production?", was addressed from two perspectives. First, the primary goal of the proposed and developed learning process was to increase the constructability and correctness of the contractor's internal design solutions. During the internal analysis of the process, it was recognized that the learning process was a valuable tool for improving the design solutions in a structured manner. Especially balancing between the personalized and codified sub-processes and implementation of the project-based community of practice (also suggested by Lin \& Lee 2012) were seen as major enablers for success.

In addition to the primary goal, the learning process is a potential enabler for tackling wider barriers for productivity development. As missing the common language of knowledge management (Mäki 2008), ignoring the deeper root causes (Dave and Koskela 2009), lack of learning from errors (Cannon \& Edmondson 2005), and forgetting to ask "how" (Eriksson et al. 2013) are hampering the development of the entire industry, the proposed learning process attempts to educate the organization to address these barriers in 
an organized manner. If the process could be implemented widely in the entire organization as a continuous process, it could enable the accelerated and cumulative learning while individuals and implemented communities of practice would internalize the culture of the continuous learning in their daily work.

The concept of continuous learning also connects the developed learning process tightly to lean principles. Giridhar et al. (2018) suggest that an effective knowledge management system can prevent 'waste of knowledge', while acting as an enabler for implementation of lean principles. Thus, it can be argued that knowledge management and lean principles are tightly intertwined when the learning processes are developed in construction organizations. However, further research is needed regarding the wider implementation and the actual, related benefits of the process, and the actual link between the proposed process and its effect on implementation of lean principles.

The design research approach taken enabled an iterative development of the process, but also the initial implementation of the process in the unit's routines, which is the first step in promoting a culture of continuous learning. The proposals of AlSehaimi et al. (2012) and Azhar (2009) were reinforced, as they encouraged to implement design research to enable the concrete development of construction processes. The approach also enabled to bridge the gap between the strategic and the operative level of actions, when the organization's wider goals were connected to the concrete actions by implementing the created process. As stated by Carrillo et al. (2013), bridging the gap is a remarkable enabler for more effective knowledge management processes, which can be seen as one of the main core competencies of construction companies in the future.

\section{CONCLUSIONS}

Even though the the relevancy of continuous learning is well recognized in construction research and within the industry, construction organizations are incapable of effectively exploiting the best practices of knowledge management. This primarily stems from the lack of mutual understanding of knowledge management terminology, no link between the knowledge management strategy and the operational processes, as well as poor ability to steer the learning processes in action. Also, construction organizations should increasingly emphasize the learning from failures, which can be enabled by implementing project-based communities of practice into organizations.

These problems were addressed by means of the iteratively developed learning process, which was able to increase the constructability and correctness of the contractor's internal design solution. In addition, the process was also seen as a potential enabler for tackling deeper root causes for ineffective knowledge management, which is an essential core competence for the construction companies. As the present research was limited to a single organization, further research could include the validation of the proposed process in different organizations, as well as in procurement and estimation units, which could also benefit from feedback received from production. Further studies could also include the management of inter-organizational learning, prevention of knowledge dispersion caused by the high mobility of workers, as well as a study on how large amounts of lessons learned information could be managed more effectively. 


\section{REFERENCES}

Almeida, M. V., \& Soares, A. L. (2014). Knowledge sharing in project-based organizations: Overcoming the informational limbo. International Journal of Information Management, 34(6), 770-779.

AlSehaimi, A., Koskela, L., \& Tzortzopoulos, P. (2012). Need for alternative research approaches in construction management: Case of delay studies. Journal of Management in Engineering, 29(4), 407-413.

Azhar, S., Ahmad, I., \& Sein, M. K. (2009). Action research as a proactive research method for construction engineering and management. Journal of Construction Engineering and Management, 136(1), 87-98.

Blackler, F. (1995). Knowledge, knowledge work and organizations: An overview and interpretation. Organization Studies, 16(6), 1021-1046.

Cannon, M. D., \& Edmondson, A. C. (2005). Failing to learn and learning to fail (intelligently): How great organizations put failure to work to innovate and improve. Long Range Planning, 38(3), 299-319.

Carrillo, P., \& Chinowsky, P. (2006). Exploiting knowledge management: The engineering and construction perspective. Journal of Management in Engineering, 22(1), 2-10.

Carrillo, P., Ruikar, K., \& Fuller, P. (2013). When will we learn? Improving lessons learned practice in construction. International Journal of Project Management, 31(4), 567-578.

Crossan, M. M., Lane, H. W., \& White, R. E. (1999). An organizational learning framework: From intuition to institution. Academy of Management Review, 24(3), 522-537.

Dave, B., \& Koskela, L. (2009). Collaborative knowledge management-A construction case study. Automation in Construction, 18(7), 894-902.

Eriksson, P. E. (2013). Exploration and exploitation in project-based organizations: Development and diffusion of knowledge at different organizational levels in construction companies. International Journal of Project Management, 31(3), 333341.

Giridhar, R. , Gaikwad, D. \& Lad, J. (2018). Knowledge Management and Its Application in Developing Lean Culture. Proceedings (IGLC 26). Chennai, India.

Hansen, M. T., Nohria, N., \& Tierney, T. (1999). What's your strategy for managing knowledge. The Knowledge Management Yearbook 2000-2001, 1-10.

Hari, S., Egbu, C. O., \& Kumar, B. (2004). Knowledge Capture in Small and Medium Enterprises in the Construction Industry: Challenges and Opportunities. 20th Annual ARCOM Conference, 2(September), 847-858.

Henderson, J. R., Ruikar, K. D., \& Dainty, A. R. J. (2013). The need to improve doubleloop learning and design-construction feedback loops: A survey of industry practice. Engineering, Construction and Architectural Management, 20(3), 290-306.

Holmström, J., Ketokivi, M., \& Hameri, A.-P. (2009). Bridging practice and theory: A design science approach. Decision Sciences, 40(1), 65-87.

Kamara, J. M., Anumba, C. J., \& Carrillo, P. M. (2002). A CLEVER approach to selecting a knowledge management strategy. International Journal of Project 
Management, 20(3), 205-211.

Kovács, G., \& Spens, K. M. (2005). Abductive reasoning in logistics research. International Journal of Physical Distribution \& Logistics Management, 35(2), 132-144

Lave, J., \& Wenger, E. (1998). Communities of practice. Retrieved June, 9, 2008.

Lee, H., \& Choi, B. (2003). Knowledge management enablers, processes, and organizational performance: An integrative view and empirical examination. Journal of Management Information Systems, 20(1), 179-228.

Lin, Y.-C., \& Lee, H.-Y. (2012). Developing project communities of practice-based knowledge management system in construction. Automation in Construction, 22, 422-432.

Nonaka, I., \& Takeuchi, H. (1995). The knowledge-creating company: How Japanese companies create the dynamics of innovation. Oxford university press.

Shook, J. (2008). Managing to learn: using the A3 management process to solve problems, gain agreement, mentor and lead. Lean Enterprise Institute.

Wang, F., \& Hannafin, M. J. (2005). Design-based research and technology-enhanced learning environments. Educational Technology Research and Development, 53(4), $5-23$.

Westerlund, B. (2007). A workshop method that involves users talking, doing and making. In Proceedings of international conference on human-machine interaction, Human07, IEEE. 